\title{
Central blood pressure variability is increased in hypertensive patients with target organ damage
}

\author{
Alejandro de la Sierra MD, PhD ${ }^{1}$ (D) | Julia Pareja MD ${ }^{1}$ | Sergi Yun MD ${ }^{1}$ | Eva Acosta MD $^{1}$ | \\ Francesco Aiello $\mathrm{MD}^{1}$ | Anna Oliveras $\mathrm{MD}, \mathrm{PhD}^{2}$ | Susana Vázquez $\mathrm{MD}^{2}$ | \\ Pedro Armario MD, PhD ${ }^{3}$ | Pedro Blanch MD ${ }^{3}$ | Cristina Sierra MD, PhD ${ }^{4}$ | \\ Francesca Calero $\mathrm{MD}^{5}$ | Patricia Fernández-Llama MD, $\mathrm{PhD}^{5}$
}

${ }^{1}$ Department of Internal Medicine, Hospital Mutua Terrassa, University of Barcelona, Terrassa, Spain

${ }^{2}$ Department of Nephrology, Hospital del Mar, Barcelona, Spain

${ }^{3}$ Cardiovascular Disease Unit, Hospital Moisés Broggi Sant Joan Despí , Spain

${ }^{4}$ Department of Internal Medicine, Hospital Clínic, Barcelona, Spain

${ }^{5}$ Renal and Hypertension Units, Fundació Puigvert Universitat Autónoma de Barcelona, Barcelona, Spain

\section{Correspondence}

Alejandro de la Sierra, MD, PhD, Department of Internal Medicine, Hospital Mutua Terrassa, University of Barcelona, Terrassa, Spain. Emails: adelasierra@mutuaterrassa.cat; asierra@ub.edu

Funding information Instituto de Salud Carlos, grant number: IIIPI14/00592.
We aimed to evaluate the association of aortic and brachial short-term blood pressure variability (BPV) with the presence of target organ damage (TOD) in hypertensive patients. One-hundred seventy-eight patients, aged $57 \pm 12$ years, 33\% women were studied. TOD was defined by the presence of left ventricular hypertrophy on echocardiogram, microalbuminuria, reduced glomerular filtration rate, or increased aortic pulse wave velocity. Aortic and brachial BPV was assessed by 24-hour ambulatory BP monitoring (Mobil-O-Graph). TOD was present in 92 patients (51.7\%). Compared to those without evidence of TOD, they had increased night-to-day ratios of systolic and diastolic BP (both aortic and brachial) and heart rate. They also had significant increased systolic BPV, as measured by both aortic and brachial daytime and 24-hours standard deviations and coefficients of variation, as well as for average real variability. Circadian patterns and short-term variability measures were very similar for aortic and brachial BP. We conclude that BPV is increased in hypertensive-related TOD. Aortic BPV does not add relevant information in comparison to brachial BPV.

\section{1 | INTRODUCTION}

Blood pressure variability (BPV) has been increasingly recognized as a prognostic factor influencing the probabilities of future cardiovascular events. ${ }^{1}$ Short-term BPV can be estimated through different parameters from 24-hour ambulatory blood pressure monitoring (ABPM). Such parameters include, the nocturnal decline in blood pressure as well as fluctuations of blood pressure during the 24-hour monitoring. Standard deviations and coefficients of variation of 24-hour, daytime, and nighttime blood pressure, as well as average real variability have been proposed as short-term variability measures with potential prognostic importance. ${ }^{2-4}$

On the other side, aortic BP seems to be a better estimator than the traditional brachial BP measurement of the "true" BP affecting target organs, such as heart, brain, and kidneys ${ }^{5}$ also showing a better predictive value of future cardiovascular events and mortality in hypertensive subjects. ${ }^{6-9}$ Measuring central BP for 24 hours has recently become possible due to technological advances. ${ }^{10}$ Thus, ABPM devices, including peripheral and central BP measurement allow for the simultaneous assessment of central and peripheral BP variability. To our knowledge, there has only been 1 previous report that evaluated central BP variability in relation with left ventricular abnormalities. ${ }^{11}$

The aim of the present study was to evaluate the association of both central and peripheral BPV with the presence of cardiac, renal, and vascular target organ damage in essential hypertensive patients.

\section{2 | PATIENTS AND METHODS}

This is a cross-sectional study, which included 178 patients aged $>18$ years, with a diagnosis of essential hypertension, mean age $57 \pm 12$ years, and $33 \%$ women who were consecutively enrolled 
from 5 hypertension units at corresponding university hospitals in the metropolitan area of Barcelona, Spain. Patients were excluded if they had confirmed or suspected secondary hypertension, or if they could not undergo 24-hour ABPM. The local institutional ethic committees approved the study protocol. Written informed consent was obtained from all participants. The investigation conforms to the principles outlined in the declaration of Helsinki.

\subsection{Measurements of blood pressure and heart rate variability}

Twenty-four hour ABPM was performed by means of a noninvasive automated oscillometric device and validated for brachial BP measurement according to the European Society of Hypertension international protocol. ${ }^{12}$ The monitor was placed on a week day between 8:00 AM and 10:00 AM, and BP was measured automatically at 20-minutes intervals throughout both the awake and asleep periods, as defined in the patients' diary. Aortic BP was estimated through brachial pulse wave analysis for each brachial BP measurement. All subjects included in the study had recordings of good technical quality (at least $80 \%$ of valid readings). Otherwise, ABPM was repeated in 1 week. The following parameters of blood pressure (both brachial and aortic) and heart rate variability were calculated, as previously reported: ${ }^{13}$

- Night-to-day ratios for systolic (SBP) and diastolic (DBP) blood pressure, as well as for heart rate (HR).

- Standard deviations (SD) and coefficients of variation (CV = SD* 100/BP) for SBP and DBP, as well as for HR for 24-hour daytime and nighttime periods.

- Weighted standard deviation (WSD) was calculated using the following formula: WSD = (daytime SD *number of hours awake) + (nighttime SD *number of hours asleep)/24.

- Average real variability (ARV) was calculated as the average of the differences (in absolute value) between consecutive BP measurements.

\subsection{Assessment of target organ damage}

Target organ damage (TOD) was defined as the presence of renal abnormalities, left ventricular hypertrophy, or arterial stiffness. Details on such assessments have been reported elsewhere. ${ }^{14}$ Briefly, renal abnormalities were defined as the presence of a reduced estimated glomerular filtration rate (eGFR), $<60 \mathrm{~mL} / \mathrm{min} / 1.73 \mathrm{~m}^{2}$ was calculated using the chronic kidney disease-epidemiology collaborative equation $^{15}$ (serum creatinine measured by an enzymatic modified Jaffe reaction) or by the presence of an urinary albumin excretion $\geq 30 \mathrm{mg} / \mathrm{g}$ of creatinine (measured by turbidimetry in local laboratories according to current recommended standards and calculated as the average of 2 spot first-morning void urine samples obtained in separate days). Left ventricular hypertrophy (LVH) was defined as a left ventricular mass index $($ LVMI $) \geq 115 \mathrm{~g} / \mathrm{m}^{2}$ in men or $\geq 95 \mathrm{~g} / \mathrm{m}^{2}$ in women was calculated by means of an echocardiography, performed according to the
American Society of Echocardiography reccomendations. ${ }^{16}$ Moreover, arterial stiffness was defined by the presence of 24-hour mean values of aortic pulse wave velocity (aPWV) $>10 \mathrm{~m} / \mathrm{s}$, calculated at each BP measurement through the Mobil-O-Graph device. ${ }^{17}$

\section{3 | Statistical analysis}

Values are expressed as means \pm standard deviations, median (interquartile range), or percentages, as needed. Student's t-test or Fisher exact test were used for the comparison between patients with and without TOD. Moreover, the association between BPV estimates and the presence of TOD was further evaluated in logistic regression models with odds ratio ( $95 \%$ confidence interval) calculation adjusted for the correspondent value of BP (24-hours, daytime, or nighttime). Pearson's or Spearman's correlation coefficients, when appropriate, were obtained to measure the association between BPV indexes and LVMI, urinary albumin excretion, eGFR, or aPWV. The SPSS for Windows version 19.0 software was used for statistical analysis.

\section{3 | RESULTS}

A total of $92(51.7 \%)$ patients had documented TOD. Left ventricular hypertrophy was present in 66 patients (37.1\%), either reduced eGFR (25) or albuminuria (31) in 47 (26.4\%), and increased aPWV in 37 (20.8\%). From them, 46 patients (50\%) presented TOD in more than 1 organ. Table 1 shows the difference in clinical parameters between patients with and without TOD.

Night-to-day ratios for SBP and DBP (both brachial and aortic) were increased in patients with TOD compared to those without. Moreover, the night-to-day ratio for heart rate was also increased in patients with TOD (Table 2).

Table 3 shows differences in SD and CV for 24-hour, day and night, brachial and aortic SBP and DBP, as well as for HR. As shown, patients with TOD had significantly higher mean values for SD for both brachial and aortic SBP, measured during 24-hour, day or night. Likewise, CV (SD corrected for absolute BP) was increased in TOD patients for both brachial and aortic SBP, measured at 24-hours or during daytime. Nighttime values were not significantly different. Heart rate variability was reduced in patients with TOD, as both SD and CV were lower during 24-hour, day and night periods.

In accordance with these results, WSD and ARV for SBP (both brachial and aortic) were also higher in patients with TOD compared to those without (Figure 1).

The logistic regression analysis (Table 4) showed that for both brachial and aortic SBP, daytime SD, WSD, and ARV were associated with TOD in unadjusted models and after adjustments for absolute BP values. In contrast, nighttime SD was only associated with TOD in unadjusted models, but not after adjustment for absolute BP. The highest odds ratio was obtained for ARV. Brachial and aortic BP showed almost identical odds ratios.

In sensitivity analyses, systolic BPV was compared separately in patients with and without LVH, kidney organ damage (either 


\begin{tabular}{|c|c|c|c|}
\hline Parameter & $\begin{array}{l}\text { With TOD } \\
(\mathrm{N}=92)\end{array}$ & $\begin{array}{l}\text { Without TOD } \\
(\mathrm{N}=86)\end{array}$ & $P$ value \\
\hline Age, y & $63.0 \pm 10.0$ & $52.5 \pm 11.5$ & $<.001$ \\
\hline Gender, \% women & 41.3 & 23.3 & .010 \\
\hline $\mathrm{BMI}, \mathrm{kg} / \mathrm{m}^{2}$ & $29.9 \pm 4.6$ & $28.8 \pm 4.5$ & .224 \\
\hline Smokers, \% & 23.9 & 24.4 & .937 \\
\hline Diabetes, \% & 26.1 & 16.3 & .018 \\
\hline Dyslipidemia, \% & 71.7 & 54.7 & .111 \\
\hline Previous cardiovascular ${ }^{\mathrm{a}}$ event, $\%$ & 29.3 & 16.3 & .039 \\
\hline Antihypertensive treatment, \% & 96.7 & 86.0 & .014 \\
\hline 24- $\mathrm{h}$ brachial SBP, mm Hg & $133.8 \pm 14.5$ & $124.5 \pm 11.0$ & $<.001$ \\
\hline 24- $\mathrm{h}$ brachial DBP, $\mathrm{mm} \mathrm{Hg}$ & $80.2 \pm 9.5$ & $79.9 \pm 7.4$ & .809 \\
\hline 24- $\mathrm{h}$ aortic SBP, $\mathrm{mm} \mathrm{Hg}$ & $122.8 \pm 15.0$ & $115.5 \pm 10.2$ & $<.001$ \\
\hline 24- $\mathrm{h}$ aortic $\mathrm{DBP}, \mathrm{mm} \mathrm{Hg}$ & $81.9 \pm 9.8$ & $81.2 \pm 7.6$ & .615 \\
\hline Daytime brachial SBP, mm Hg & $136.9 \pm 15.1$ & $128.9 \pm 12.1$ & $<.001$ \\
\hline Daytime brachial DBP, $\mathrm{mm} \mathrm{Hg}$ & $83.5 \pm 10.3$ & $84.0 \pm 8.2$ & .726 \\
\hline Daytime aortic SBP, mm Hg & $125.9 \pm 14.8$ & $118.9 \pm 10.6$ & $<.001$ \\
\hline Daytime aortic DBP, mm Hg & $85.5 \pm 10.6$ & $85.6 \pm 8.5$ & .918 \\
\hline Night brachial SBP, mm Hg & $126.8 \pm 17.3$ & $115.7 \pm 12.4$ & $<.001$ \\
\hline Night brachial DBP, mm Hg & $73.8 \pm 9.9$ & $71.3 \pm 8.8$ & .057 \\
\hline Night aortic SBP, mm Hg & $17.3 \pm 18.8$ & $108.3 \pm 12.4$ & $<.001$ \\
\hline Night aortic DBP, mm Hg & $74.8 \pm 10.3$ & $72.3 \pm 9.0$ & .055 \\
\hline eGFR, $\mathrm{mL} / \mathrm{min} / 1.73 \mathrm{~m}^{2}$ & $73.7 \pm 20.5$ & $90.6 \pm 15.0$ & $<.001$ \\
\hline Urinary albumin excretion, $\mathrm{mg} / \mathrm{g}$ & $13[4.9-112]$ & $4.8[2.5-9.4]$ & $<.001$ \\
\hline LVMI, g/m² & $116.8 \pm 27.7$ & $84.7 \pm 16.4$ & $<.001$ \\
\hline 24-h aPWV, m/s & $9.3 \pm 1.6$ & $7.6 \pm 1.2$ & $<.001$ \\
\hline
\end{tabular}

TABLE 1 Differences in clinical parameters between patients with and without target organ damage (TOD)

aPWV, aortic pulse wave velocity; BMI, body mass index; DBP, diastolic BP; eGFR, estimated glomerular filtration rate; LVMI, left ventricular mass index; SBP, systolic BP.

${ }^{a}$ Means cardiac or cerebrovascular event.

\begin{tabular}{|llll|}
\hline Parameter & $\begin{array}{l}\text { With TOD } \\
(\mathbf{N}=92)\end{array}$ & $\begin{array}{l}\text { Without TOD } \\
(\mathbf{N}=86)\end{array}$ & P value \\
\hline Brachial & & & \\
\hline Nigh-to-day ratio for SBP, \% & $93.0 \pm 8.7$ & $90.1 \pm 8.1$ & .023 \\
\hline Nigh-to-day ratio for DBP, \% & $89.2 \pm 9.5$ & $85.4 \pm 10.1$ & .012 \\
\hline Aortic & & & \\
\hline Nigh-to-day ratio for SBP, \% & $94.2 \pm 9.0$ & $91.3 \pm 8.7$ & .028 \\
\hline Nigh-to-day ratio for DBP, \% & $88.5 \pm 9.2$ & $84.6 \pm 9.9$ & .008 \\
\hline Heart rate & & & \\
\hline Night-to-day ratio, \% & $88.9 \pm 9.2$ & $84.1 \pm 10.7$ & .002 \\
\hline
\end{tabular}

TABLE 2 Night-to-day ratios for brachial and aortic systolic and diastolic BP, and heart rate in patients with or without target organ damage (TOD)

DBP, diastolic BP; SBP, systolic BP.

albuminuria or reduced eGFR, albuminuria only or reduced eGFR only), or aortic stiffness (Tables S1-S5 at supplemental digital content). All the groups with specific alterations had increased values of both brachial and aortic WSD, ARV, and daytime SD $(P<.05$ for all comparisons). Nighttime SD was also increased in patients with aortic stiffness and reduced renal function, but not in those with microalbuminuria or LVH.

Finally, the association between TOD and BPV was also assessed by calculating Pearson's or Spearman's correlation coefficients between systolic BPV parameters and either LVMI, urinary albumin 
TABLE 3 Standard deviation and coefficient of variation for brachial and aortic blood pressures, as well as for heart rate in patients with or without target organ damage (TOD)

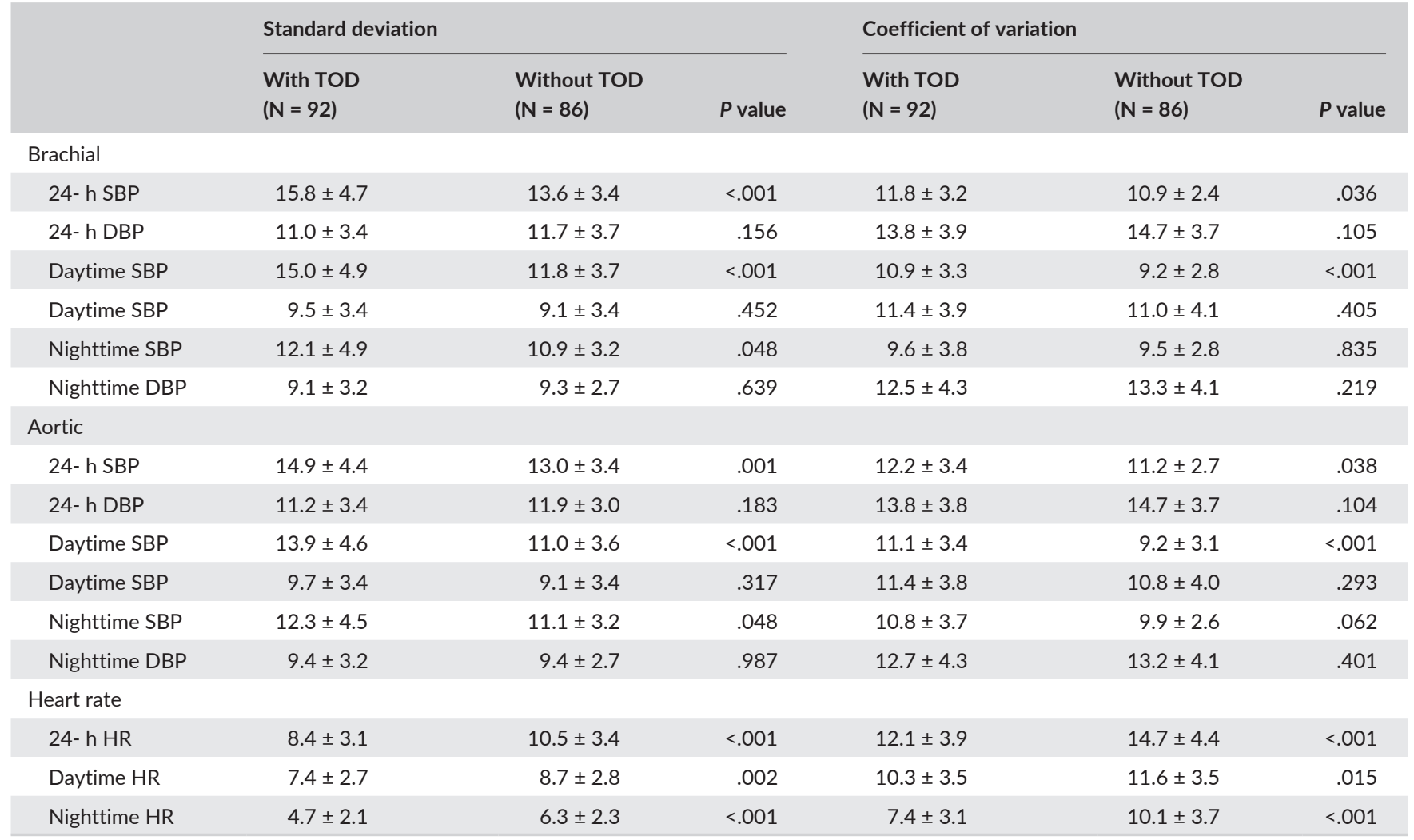

DBP, diastolic BP; HR, heart rate; SBP, systolic BP.

FIGURE 1 Comparison of weighted standard deviation (WSD; left panel) and average real variability (AVR; right panel) for brachial (B) and aortic (A) systolic (SBP) and diastolic (DBP) blood pressure between patients with (dashed bars) or without (open bars) target organ damage. ${ }^{*}$ indicates $P<.001$ in the comparison between groups

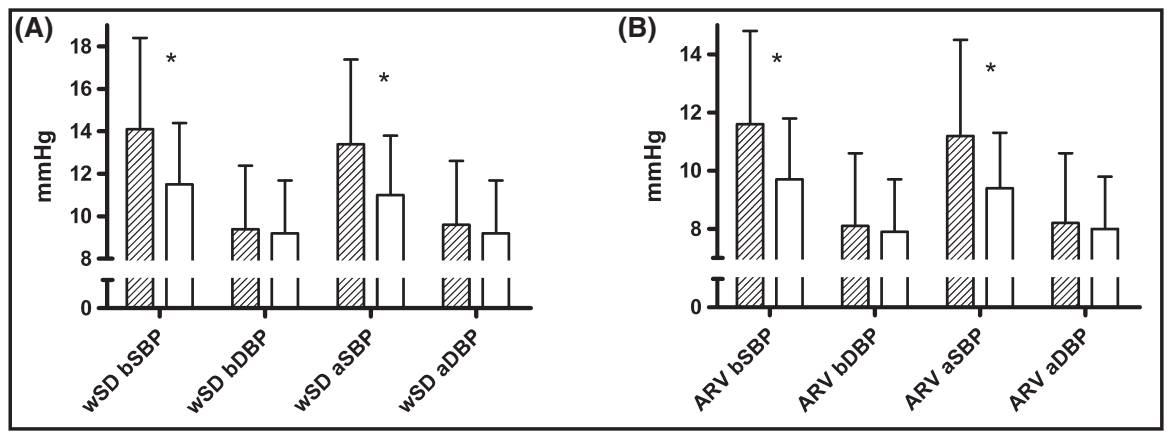

excretion, eGFR, or aPWV (Table 5). Such analyses also revealed a significant correlation of both brachial and aortic WSD, ARV, and daytime SD with all markers of organ damage. The significance was extended to night SD and coefficients of variation for aPWV, and in some parameters to urinary albumin excretion and eGFR.

\section{4 | DISCUSSION}

This study shows that patients with hypertensive-related organ damage, either at cardiac, renal, or vascular levels present an increased short-term systolic BPV. Both brachial and aortic BPV show the same pattern, which includes a blunted nocturnal BP decline, increased daytime and weighted 24-hour standard deviations, and increased average real variability. All such relationships remain significant after absolute BP adjustments and are evident in sensitivity analyses in separated groups with LVH, either reduced eGFR or microalbuminuria, or increased aortic stiffness.

Increased BPV is considered a risk factor for cardiovascular mortality and morbidity. ${ }^{1}$ Long-term or mid-term BPV can be measured by routine office $B P$, including seasonal variations and visit-to-visit differences. ABPM is useful for estimating short-term BPV, which includes circadian changes as well as differences between consecutive measures. Several estimates of short-term BPV have been associated with the degree of target organ damage, either at cardiac or vascular levels, whereas the relationship with kidney damage has been less studied. A recent meta-analysis of 12 previous studies found a correlation between daytime and weighted standard deviations, as well as ARV 
TABLE 4 Odds ratios $(95 \% \mathrm{Cl})$ of the association with target organ damage as dependent variable for daytime, nighttime and weighted standard deviations, and for average real variability (for each $\mathrm{mm} \mathrm{Hg}$ increase) of systolic brachial and aortic blood pressure before and after adjustment for the corresponding BP absolute values

\begin{tabular}{|c|c|c|}
\hline Parameter & Unadjusted & $\begin{array}{l}\text { Adjusted for } 24-\mathrm{h} \text {, } \\
\text { day, or night BP }\end{array}$ \\
\hline \multicolumn{3}{|l|}{ Brachial } \\
\hline Daytime SD & $1.21(1.11-1.32)$ & $1.17(1.07-1.28)$ \\
\hline Nighttime SD & $1.08(1.00-1.16)$ & $1.04(0.96-1.12)$ \\
\hline Weighted SD & $1.25(1.12-1.38)$ & $1.17(1.05-1,30)$ \\
\hline ARV & $1.31(1.16-1.49)$ & $1.21(1.06-1.39)$ \\
\hline \multicolumn{3}{|l|}{ Aortic } \\
\hline Daytime SD & $1.22(1.11-1.34)$ & $1.18(1.08-1.30)$ \\
\hline Nighttime SD & $1.08(1.00-1.17)$ & $1.05(0.97-1.14)$ \\
\hline Weighted SD & $1.26(1.13-1.41)$ & $1.83(1.30-1.35)$ \\
\hline ARV & $1.30(1.14-1.48)$ & $1.26(1.10-1.44)$ \\
\hline
\end{tabular}

ARV, average real variability; SD, standard deviation.

Target organ damage considered when the patient presented either left ventricular hypertrophy, renal damage (either reduced estimated glomerular filtration rate or albuminuria), or increased aortic pulse wave velocity.

with left ventricular mass index. ${ }^{18}$ Moreover, short-term BPV has also been correlated with the degree of aortic stiffness. ${ }^{19}$ In contrast, the association with kidney damage has not been fully confirmed. Indeed, in a study with subjects participating in the Jackson Heart Study, the increased BPV observed in patients with either microalbuminuria, or reduced eGFR was no longer significant after adjusting for the absolute level of BP. ${ }^{20}$

Our results confirm that patients with hypertensive-related cardiac, vascular, or renal damage have an increased systolic BPV. These differences are present with different estimators, including daytime and 24-hour SD and CV as well as ARV, a marker of fluctuations between consecutive measures. Moreover, we did not detect differences that were dependent on the organ affected, with patients with LVH, kidney damage, or aortic stiffness showing the same pattern. In addition, the increased BPV was independent of the degree of BP elevation, as adjusted odds ratios were statistically significant.

On the other hand, central (aortic) blood pressure has been recognized as a better estimator of "true" BP directly transmitted to the heart, brain, and kidneys in comparison to peripheral (brachial) BP. ${ }^{5}$ Central BP has been found to be better correlated with cardiac and vascular organ damage ${ }^{21}$ in comparison to peripheral BP, although its incremental value with respect to brachial BP has not been fully assessed. With respect to 24-hour estimators of central BP, 1 study found superiority over peripheral BP in the correlation with left ventricular mass index. ${ }^{22}$ However, we have previously found that both central and peripheral BP were similarly associated with TOD (cardiac, renal, or vascular), and according to our results, the association of 24-hour central BP was no longer significant after adjusting for peripheral BP. ${ }^{14}$
TABLE 5 Correlation coefficients of the relationship between brachial and aortic systolic blood pressure variability and left ventricular mass index (LVMI), urinary albumin excretion (UAE), estimated glomerular filtration rate (eGFR) and aortic pulse wave velocity (aPWV)

\begin{tabular}{|c|c|c|c|c|}
\hline Parameter & LVMI & $U A E^{a}$ & eGFR & aPWV \\
\hline \multicolumn{5}{|l|}{ Brachial } \\
\hline 24- h standard deviation & .137 & $.238^{*}$ & $-.203^{*}$ & $.367^{*}$ \\
\hline Day standard deviation & $.232^{*}$ & $.209^{*}$ & $-.234^{*}$ & $.432^{*}$ \\
\hline Night standard deviation & .117 & $.250^{*}$ & $-.234^{*}$ & $.432^{*}$ \\
\hline $\begin{array}{l}\text { 24- } \mathrm{h} \text { coefficient of } \\
\text { variation }\end{array}$ & .033 & .142 & -.074 & $.291^{*}$ \\
\hline $\begin{array}{l}\text { Day coefficient of } \\
\text { variation }\end{array}$ & $.157^{*}$ & .125 & $-.203^{*}$ & $.221^{*}$ \\
\hline $\begin{array}{l}\text { Night coefficient of } \\
\text { variation }\end{array}$ & .001 & $.167^{*}$ & $-.246^{*}$ & $.326^{*}$ \\
\hline $\begin{array}{l}\text { Weighted standard } \\
\text { deviation }\end{array}$ & $.231^{*}$ & $.241^{*}$ & $-.218^{*}$ & $.453^{*}$ \\
\hline Average real variability & $.214^{*}$ & $.217^{*}$ & $-.205^{*}$ & $.445^{*}$ \\
\hline \multicolumn{5}{|l|}{ Aortic } \\
\hline 24- h standard deviation & .105 & $.197^{*}$ & $-.197^{*}$ & $.387^{*}$ \\
\hline Day standard deviation & $.201^{*}$ & $.193^{*}$ & $-.237^{*}$ & $.439^{*}$ \\
\hline Night standard deviation & .125 & $.200^{*}$ & -.084 & $.326^{*}$ \\
\hline $\begin{array}{l}\text { 24-h coefficient of } \\
\text { variation }\end{array}$ & .023 & .115 & $-.189^{*}$ & $.268^{*}$ \\
\hline $\begin{array}{l}\text { Day coefficient of } \\
\text { variation }\end{array}$ & .139 & .127 & $-.243^{*}$ & $.348^{*}$ \\
\hline $\begin{array}{l}\text { Night coefficient of } \\
\text { variation }\end{array}$ & .073 & $.161^{*}$ & -.077 & $.225^{*}$ \\
\hline $\begin{array}{l}\text { Weighted standard } \\
\text { deviation }\end{array}$ & $.208^{*}$ & $.226^{*}$ & $-.225^{*}$ & $.472^{*}$ \\
\hline Average real variability & $.159^{*}$ & $.193^{*}$ & $-.170^{*}$ & $.431^{*}$ \\
\hline
\end{tabular}

${ }^{a}$ Correlation with UAE analyzed by Spearman's correlation coefficient. Other correlations by Pearson's correlation coefficient. ${ }^{*} P<.05$.

In the current paper, the results regarding aortic BP-variability followed the same pattern observed with absolute BP values. Several indexes of aortic BPV were associated with TOD considered as a whole, or in separated groups of patients with LVH, kidney damage, or increased aortic PWV. These included almost all indexes of systolic BPV, such as daytime and time-weighted SD, CV, and ARV. However, the observed pattern was identical to what was observed with brachial BPV. Mean values of SD, CV, ARV, as well as nocturnal BP dip were very similar for brachial and aortic estimates. Differences between patients with or without TOD or odds ratio for unadjusted or adjusted associations were almost identical.

To our knowledge, there is only 1 report examining the association of short-term central BPV with cardiac organ damage, as determined by LVH or diastolic dysfunction. ${ }^{11}$ In it, both brachial and aortic systolic BPV estimates were associated with cardiac damage. The authors claimed that central BPV was superior to peripheral BPV, because in the multiple adjustments only aortic ARV, but not brachial ARV, or 
aortic or brachial weighted SD, remained significant. Our results are conceptualize that aortic BPV and brachial BPV correlate with TODnot only cardiac, but also renal or vascular damage. However, we were unable to detect any difference between aortic and brachial systolic BPV estimates. Based on our results, we cannot confirm that central BPV measurement has advantages with respect to traditional peripheral BPV.

The discrepancy between our results and those provided by Chi et al $^{11}$ are probably due to differences in the severity of patients $(37 \%$ of our patients had LVH whereas it was present in only $8 \%$ of patients in the aforementioned study) and to differences in the methodological analysis. In fact, in the results reported by $\mathrm{Chi}$ et al ${ }^{11}$ correlation coefficients were almost identical for brachial and aortic BPV. Only in the multivariate analysis, aortic ARV, but not aortic WSD retained statistical significance (none of these parameters at brachial level remained significant). We think that such analyses in a group of patients with a very low proportion of the LVH phenotype can be easily influenced by other variable distribution. We think that this is weak evidence to support the superiority of aortic over brachial BPV.

This study has its limitations. First, its cross-sectional nature allows only descriptive associations, but it does not explore the predictive value of central or peripheral BPV in the development, progression, or regression of target organ damage. Second, most patients included were hypertensives of long duration currently on antihypertensive treatment and often with other co-morbid conditions, such as diabetes and lipid disorders, all circumstances that can influence both TOD and BPV.

In conclusion, hypertensive patients with target organ damage exhibited a blunted nocturnal BP dip and higher systolic BPV values than those without such organ damage. This elevation in BPV is manifested by assessing several estimates, including 24-hour and daytime SD and CV, as well as ARV. Both brachial and aortic BPV show the same pattern of a blunted nocturnal decline and increased systolic BPV. According to our results, calculations of aortic BPV do not seem to add any advantage over brachial BPV.

\section{ACKNOWLEDGMENTS}

Supported in part by the Instituto de Salud Carlos III (PI14/00592), and by Fundació de Recerca I Docència Mútua Terrassa.

\section{CONFLICT OF INTEREST}

The authors declare no conflicts of interest in relation to this paper.

\section{ORCID}

Alejandro de la Sierra iD http://orcid.org/0000-0002-6548-5497

\section{REFERENCES}

1. Parati G, Ochoa JE, Lombardi C, Bilo G. Assessment and management of blood-pressure variability. Nat Rev Cardiol. 2013;10:143-155.
2. Bilo G, Giglio A, Styczkiewicz K, et al. A new method for assessing 24-h blood pressure variability after excluding the contribution of nocturnal blood pressure fall. J Hypertens. 2007;25:2058-2066.

3. Mena L, Pintos S, Queipo NV, Aizpúrua JA, Maestre G, Sulbarán T. A reliable index for the prognostic significance of blood pressure variability. J Hypertens. 2005;23:505-511.

4. Hansen TW, Thijs L, Li Y, et al. Prognostic value of reading-to-reading blood pressure variability over 24 hours in 8938 subjects from 11 populations. Hypertension. 2010;55:1049-1057.

5. McEniery CM, Cockroft JR, Roman MJ, Franklin SS, Wilkinson IB. Central blood pressure: current evidence and clinical importance. Eur Heart J. 2014;35:1719-1725.

6. Roman MJ, Devereux RB, Kizer JR, et al. Central pressure more strongly relates to vascular disease and outcome than does brachial pressure: the Strong Heart Study. Hypertension. 2007;50: 197-203.

7. Williams B, Lacy PS, Thom SM, et al. Differential impact of blood pressure-lowering drugs on central aortic pressure and clinical outcomes: principal results of the Conduit Artery Function Evaluation (CAFE) study. Circulation. 2006;113:1213-1225.

8. Mitchell GF, Hwang SJ, Vasan RS, et al. Arterial stiffness and cardiovascular events: the Framingham Heart Study. Circulation. 2010;121:505-511.

9. Vlachopoulos C, Aznaouridis K, O'Rourke MF, Safar ME, Baou K, Stefanadis C. Prediction of cardiovascular events and all-cause mortality with central haemodynamics: a systematic review and metaanalysis. Eur Heart J. 2010;31:1865-1871.

10. Stergiou GS, Parati G, Vlachopoulos C, et al. Methodology and technology for peripheral and central blood pressure and blood pressure variability measurement: current status and future directions - position statement of the European Society of Hypertension Working Group on blood pressure monitoring and cardiovascular variability. J Hypertens. 2016;34:1665-1677.

11. Chi C, Yu SK, Auckle R, et al. Association of left ventricular structural and functional abnormalities with aortic and brachial blood pressure variability in hypertensive patients: the SAFAR study. J Human Hypertens. 2017;31:633-639.

12. Franssen PM, Imholz BP. Evaluation of the Mobil-O-Graph new generation ABPM device using the ESH criteria. Blood Press Monit. 2010;15:225-228.

13. De la Sierra A, Pareja J, Armario P, et al. Renal denervation vs. spironolactone in resistant hypertension: effects on circadian patterns and blood pressure variability. Am J Hypertens. 2017;30:37-41.

14. De la Sierra A, Pareja J, Fernández-Llama P, et al. Twenty-four-hour central blood pressure is not better associated with hypertensive target organ damage than 24-h peripheral blood pressure. J Hypertens. 2017:35:2000-2005

15. Levey AS, Stevens LA, Schmid $\mathrm{CH}$, et al. A new equation to estimate glomerular filtration rate. Ann Intern Med. 2009;150:604-612.

16. Marwick TH, Gillebert TC, Aurigemma G, et al. Recommendations on the use of echocardiography in adult hypertension: a report from the European Association of Cardiovascular Imaging (EACVI) and the American Society of Echocardiography (ASE). J Am Soc Echocardiogr. 2015;28:727-754.

17. Hametner B, Wassertheurer S, Kropf J, Mayer C, Eber B, Weber T. Oscillometric estimation of aortic pulse wave velocity: comparison with intra-aortic catheter measurements. Blood Press Monit. 2013;18:173-176

18. Madden JM, O'Flynn AM, Fitzgerald AP, Kearney PM. Correlation between short-term blood pressure variability and left-ventricular mass index: a meta-analysis. Hypertens Res. 2016;39:171-177.

19. Schillaci G, Bilo G, Pucci G, et al. Relationship between short-term blood pressure variability and large-artery stiffness in human hypertension: findings from 2 large databases. Hypertension. 2012;60: 369-377. 
20. Tanner RM, Shimbo D, Dreisbach AW, Carson AP, Fox ER, Muntner P. Association between 24-hour blood pressure variability and chronic kidney disease: a cross-sectional analysis of African Americans participating in the Jackson heart study. BMC Nephrol. 2015;16:84

21. Kollias A, Lagou S, Zeniodi ME, Boubouchairpoulou N, Stergiou GS. Association of central versus brachial blood pressure with targetorgan damage. Systematic review and meta-analysis. Hypertension. 2016;67:183-190.

22. Protogerou AD, Argyris AA, Papaioannou TG, et al. Left-ventricular hypertrophy is associated better with 24-h aortic pressure than 24-h brachial pressure in hypertensive patients: the SAFAR study. J Hypertens. 2014;32:1805-1814.

\section{SUPPORTING INFORMATION}

Additional Supporting Information may be found online in the supporting information tab for this article.

How to cite this article: de la Sierra A, Pareja J, Yun S, et al. Central blood pressure variability is increased in hypertensive patients with target organ damage. J Clin Hypertens. 2018;20:266-272. https://doi.org/10.1111/jch.13172 